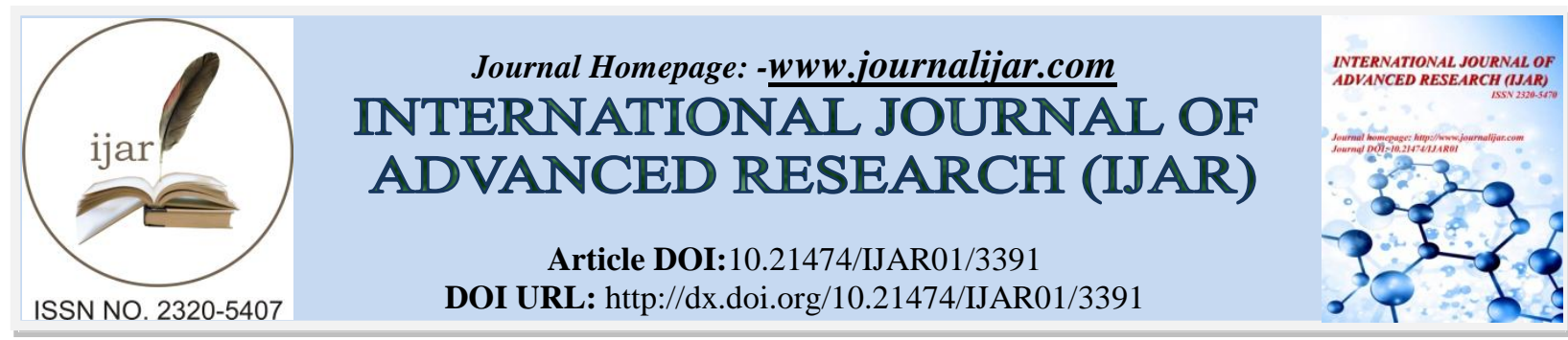

RESEARCH ARTICLE

\title{
PANCREATIC CANCER IN THE YOUNG PRESENTING AS FEVER OF UNKNOWN ORIGIN
}

\author{
Dr.Ali Al-Amri ${ }^{1}$ and Dr. Sarah M. Al-hajri ${ }^{2}$. \\ 1. Oncology Associate consultant professor at Imam Abdulrahman Bin Faisal University -Dammam. \\ 2. Medical Intern at Imam Abdulrahman Bin Faisal University-Dammam.
}

\section{Manuscript Info}

Manuscript History

Received: 22 December 2016

Final Accepted: 26 January 2017

Published: February 2017

\section{Abstract}

Pancreatic cancer is a lethal disease, presenting in the old age. We describe a rare case of a young male patient presenting with fever of unknown origin and found to have pancreatic cancer. Upon review of literature, only one case was reported.

Copy Right, IJAR, 2017,. All rights reserved.

\section{Introduction: -}

Pancreatic cancer is one of the most notorious diseases. It ranks as the $4^{\text {th }}$ cause of cancer-related death. In the US, in 2009, an estimated 42,470 new patients were diagnosed with pancreatic cancer (21,050 men and 21,420 women), with 35,240 deaths from it (18,030 men and 17,210 women). ${ }^{[1]}$ Between 2002 and 2006, the median age at diagnosis was 72 years and the median age at death was 73 years for cancer of the pancreas. The age-adjusted incidence rate in this period was 13.1 per 100,000 men and 10.4 per 100,000 women per year. ${ }^{[2], ~}{ }^{[3]}$ In Saudi Arabia, pancreatic cancer represents $1.75 \%$ of all cancers with 1585 registered cases from 1994 to 2007 accounting $2.5 \%$ for males and $1.1 \%$ for females of all types of cancer and it is the fifth most prevalent gastrointestinal cancer among Saudis. The median age at diagnosis was 63-year-old ranging from 15-96 years. [4] The most recognizable risk factor for pancreatic cancer was cigarette smoking. Hereditary pancreatic cancer occurs in 5-10\% of cases and it occurs at earlier age and usually associated with other syndromes like hereditary pancreatitis, Peutz-Jeghers syndrome, familial atypical multiple mole melanoma, familial breast cancer syndrome, and hereditary non-polyposis colorectal cancer syndrome.

Pancreatic adenocarcinoma is the most common pathological type and it's $80 \%$ located in the head of the pancreas. It usually presents with jaundice, abdominal pain, anorexia, cachexia, new onset diabetes mellitus and venous thromboembolism. Fever as the initial presentation is rare and scarcely described in literature. Therefore, we here report a case of pancreatic adenocarcinoma diagnosed in a young patient and presenting as fever at time of diagnosis.

\section{Case Report: -}

We report a case of a 29 year-old male who works as an office clerk in Ministry of Health, who presented to the ED of King Fahad University Hospital (KFUH) with history of fever $\left(39^{\circ} \mathrm{C}\right)$, anorexia, weight loss of $7 \mathrm{Kg}$, RUQ abdominal pain and jaundice for 6 weeks. He denied history of diarrhea and arthralgia.

On physical examination, a young, malnourished gentleman lying in bed, conscious and alert, apparently looking jaundiced and pale. Temperature was $39^{\circ} \mathrm{c}$ at presentation with no chills. Abdominal examination revealed hepatomegaly (16 cm liver span), however, no splenomegaly or ascites. 
The patient has no significant past medical history except for cigarette smoking 1 pack/year for the past 10 years. No history of contact with febrile patient, alcohol consumption, blood transfusion, IV drug abuse or recent history of travel. There was only a brief exposure to cattle in a farm but no raw milk ingestion. No personal or family history of cancers.

Patient was extensively investigated. Laboratory work up results are summarized in Table 1.

\begin{tabular}{|l|l|}
\hline Test & Result \\
\hline Complete Blood Count & Leukocytosis: $11.1 \times 10^{3}$ cells/mm \\
& Neutrophilic predominance $79 \%$ \\
& Hemoglobin: $10.8 \mathrm{~g} / \mathrm{dL}$ \\
& MCV: $80.6 \mathrm{fL}$ \\
\hline Liver function Test, Amylase and & Hyperbilirubinemia: Total bilirubin5 $\mathrm{mg} / \mathrm{dL}$, direct bilirubin4.3 mg/dL \\
Lipase & Albumin: $2 \mathrm{~g} / \mathrm{dL}$ \\
& Lactate dehydrogenase: $1141 \mathrm{U} / \mathrm{L}$ \\
& Gamma-gultamyltransferase:381 U/L \\
& Alkaline phosphatase:334 U/L \\
& Aspartate aminotransferase: $83 \mathrm{U} / \mathrm{L}$ \\
& Alanine aminotransferase: $35 \mathrm{U} / \mathrm{L}$ \\
& Amylase: 27 U/L \\
& Lipase: 123 U/L \\
\hline Septic work up & Repeated blood culture: no growth. \\
& Malaria smear, hepatitis profile, monospot test, EBV serology, HIV serology \\
& and echinococcus serology all were negative. \\
& Brucella serology was $1: 320$ \\
\hline Inflammatory markers & ESR: 120 h/mm \\
& CRP: 21.1 mg /L \\
\hline Autoimmune markers & ANA, ANCA, RF,AMA and ASMA* all were negative. \\
\hline Cancer markers & CA 19-9: 2289 \\
& AFP and CEA not elevated \\
\hline Random Blood Sugar & 93 mg/dL \\
\hline
\end{tabular}

* ANA: Antinuclear Antibody, ANCA: Antinuclear cytoplasmic antibody, RF: Rheumoatoid factor, AMA: Antimitochondrial antibody and ASMA: Antismooth muscle antibody.

Abdominal ultrasonography revealed multiple hypoechoic lesions in the liver and hepatomegaly. Gallbladder, kidneys were normal and no free fluid collection in the abdomen (Figure 1). A computed topography (CT) scan with contrast was requested and revealed bilateral cervical lymphadenopathy, hepatomegaly and multiple hypodense lesions of variable size and density involving both right and left hepatic lobes (Figure 2).Also, a necrotic, cystic mass lesion was noted in the head of the pancreas, measuring $3 \times 2.8 \times 2.4 \mathrm{~cm}$ in its transverse, antero-posterior and cranio-caudal dimensions, there is adjacent necrotic lymph node in the pancreatic bed (Figure 3).No splenomegaly or ascites noted.

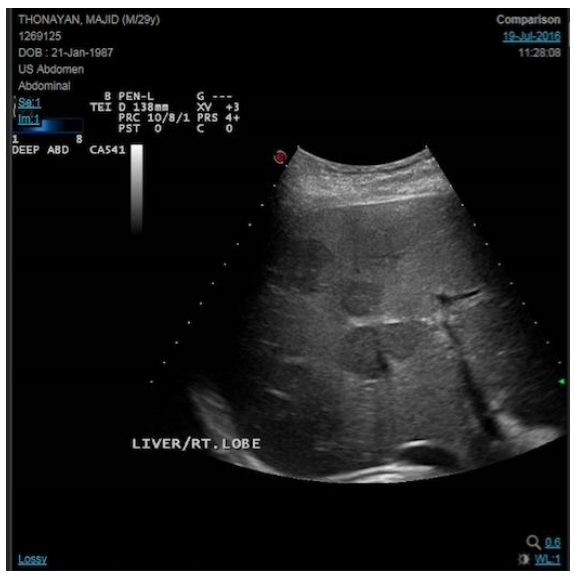

Figure 1:- US showing multiple hypoechoic lesions in the liver. 


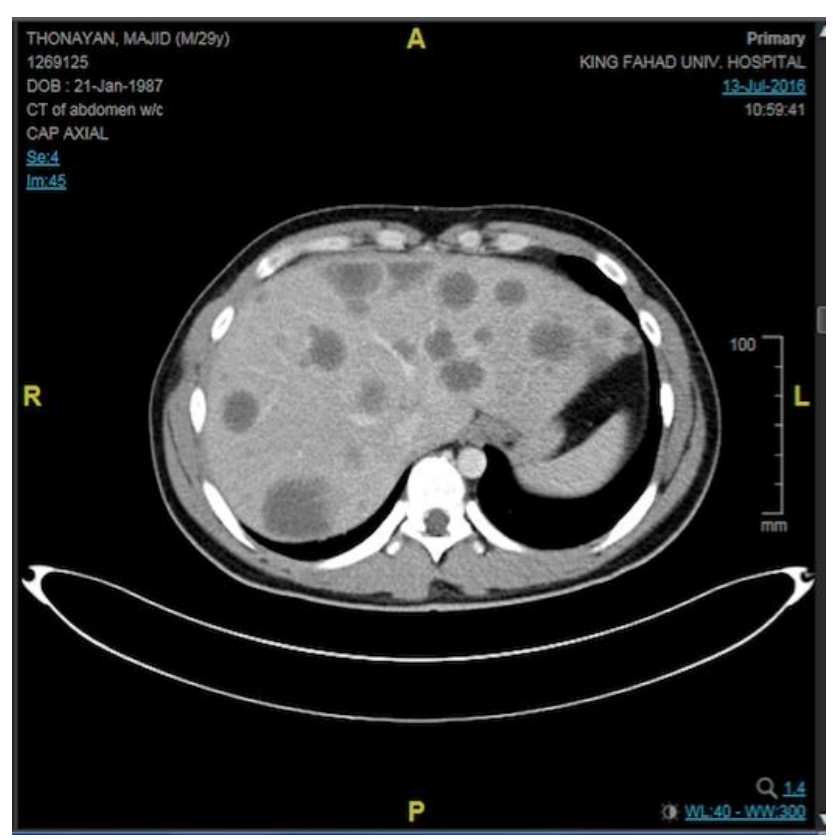

Figure 2:- CT scan showing multiple Hypodense lesions in the liver.

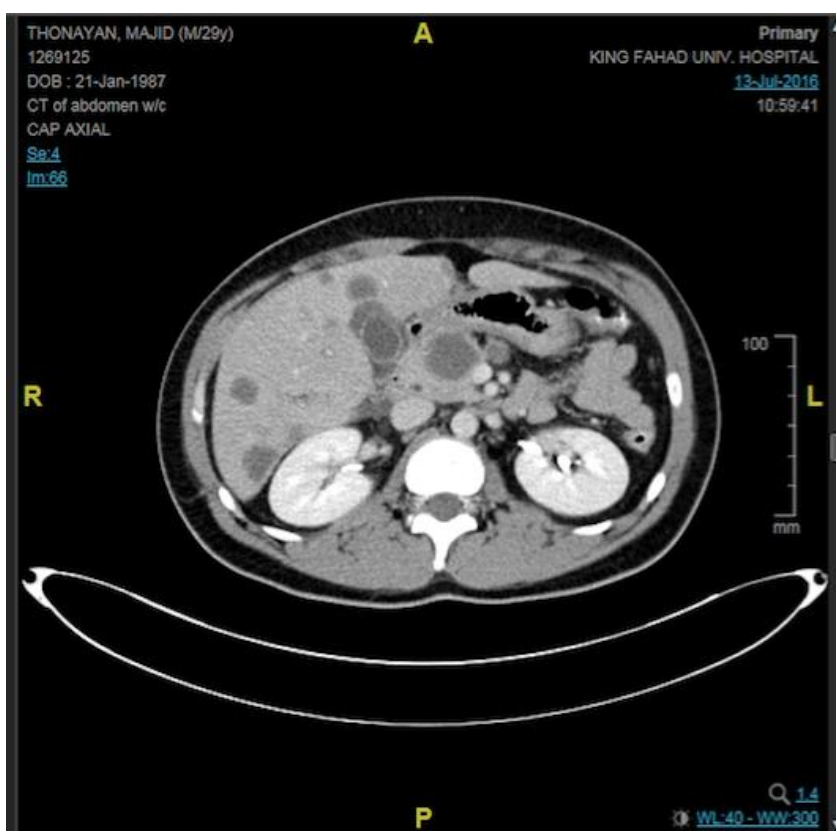

Figure 3:- CT scan showing mass in the pancreatic head.

The case was approached by a multidisciplinary team involving Infectious disease team and Gastroenterology team. Initial management plan was to start the patient on antibiotic regimen for brucellosis, Doxycycline and rifampicin were given for 6 weeks with no improvement of symptoms. A suspicion of malignancy leadthe team to carry out an ultrasound- guided liver biopsy and it revealed multiple metastatic clusters and cords of anaplastic malignant epithelial cells dispersed in a desmoplastic stroma. Extensive tumor necrosis was noted.

Oncology team was consulted and assessed the case. The treatment suggested was perusing palliative care with analgesia and chemotherapy in the form of gemcitabine and erlotinib, which is not available at our hospital. Patient was referred to a tertiary care hospital for perusing chemotherapy. 


\section{Discussion: -}

Fever of unknown origin (FUO) is defines as temperature $\geq 38.5^{\circ} \mathrm{c}$ for $\geq 3 \mathrm{wks}$ in a healthy immunocompetent individual despite a 1-week extensive investigation. Infection accounts for the most cases of FUO, followed by neoplastic fever. Neoplastic fever is a common presentation of certain malignancies like hematological, colon and renal cancers. Neoplastic fever is noted especially in metastatic malignancies. [5] Therefore, we report our rare case of a young patient presenting with fever as the main presentation of pancreatic cancer.

There are multiple mechanisms to explain fever in setting of malignancy. Infection is the usual precipitants, however, neoplastic fever can be induced by the tumor related release of pro-inflammatory cytokines. Also, aggressive body immunological response mounted against tumor with leukocytosis add to the production of pyrogens.

Pancreatic cancer is a disease of the old population. Early onset pancreatic cancer (EOPC) is defined as pancreatic cancer in $\leq 50$ years of age. Several risk factors have been linked to the susceptibility for developing pancreatic cancer at a younger age, among them, smoking and genetic factors were the key players. In a case control study conducted by Hassan MM et al., there was a $60 \%$ greater risk among the ever-smokers. [6]

EOPC usually presents as a poorly differentiated adenocarcinoma with distant metastases. In A retrospective analysis of consecutive cases of patients with EOPC, $61 \%$ had poorly differentiated pathology and $84 \%$ had metastatic unrespectable disease at time of diagnosis. [7]

Pancreatic adenocarcinoma in the young carries a poor prognosis with average survival as short as 5 months unfortunately. The patient is offered a palliative care in the form of analgesia and chemotherapy.

\section{Conclusion: -}

We conclude that pancreatic adenocarcinoma can present in earlier age with smoking and genetic susceptibility being the most critical risk factors. In addition, we found that pancreatic adenocarcinoma can present with fever of unknown origin.

\section{References: -}

1. Jemal A, Siegel R, Ward E, et al. Cancer statistics, 2009. CA Cancer J Clin. 2009;59:225-249.

2. National Cancer Institute. SEER cancer statistics review, 1975-2006, based on November 2008 SEER data submission. 2009. http://seer.cancer.gov/

3. Hariharan D, Saied A, Kocher HM. Analysis of mortality rates for pancreatic cancer across the world. HPB (Oxford). 2008;10:58-62

4. Pancreatic cancer in Saudi patients treated at tertiaryinstitution,Ten years retrospective studyHamza J. AlGhamdi, MBBS, Salem A. Alfaifi, MBBS, AshwaqA.Alolayan, MD, Salma MA. Musaad, MD, PhD,AbdulRahman M. Jazieh, MD, MPH.

5. Pancreatic carcinoma masked as feverof unknown origin, A case report and comprehensive review of literature.Ning Shi, MDa, Cheng Xing, MDa, Xiaoyan Chang, MDb, Menghua Dai, MDa,*, Yupei Zhao, MdaRisk factors for pancreatic cancer: case-control study.

6. Hassan $\mathrm{MM}^{1}$, Bondy ML, Wolff RA, Abbruzzese JL, Vauthey JN, Pisters PW, Evans DB, Khan R, Chou TH, Lenzi R, Jiao L, Li D.

7. Early onset pancreatic cancer: risk factors, presentation and outcome.

8. Piciucchi $\mathrm{M}^{1}$, Capurso $\mathrm{G}^{1}$, Valente $\mathrm{R}^{1}$, Larghi $\mathrm{A}^{2}$, Archibugi $\mathrm{L}^{1}$, Signoretti $\mathrm{M}^{1}$, Stigliano $\mathrm{S}^{1}$, Zerboni $\mathrm{G}^{1}$, Barucca $\mathrm{V}^{3}$, La Torre $\mathrm{M}^{4}$, Cavallini $\mathrm{M}^{4}$, Costamagna $\mathrm{G}^{2}$, Marchetti $\mathrm{P}^{3}$, Ziparo $\mathrm{V}^{4}$, DelleFave $\mathrm{G}^{5}$. 